\title{
Phosphorylation profiles of protein kinases in alveolar and embryonal rhabdomyosarcoma
}

\author{
Ling Cen ${ }^{1,2}$, Kristy J Arnoczky ${ }^{3}$, Fu-Chuan Hsieh ${ }^{1}$, Huey-Jen Lin ${ }^{4}$, Stephen J Qualman ${ }^{1,3}$, \\ Songlin $\mathrm{Yu}^{5}$, Huiyun Xiang ${ }^{5}$ and Jiayuh $\operatorname{Lin}^{1,2,3,6}$ \\ ${ }^{1}$ Department of Pediatrics, Center for Childhood Cancer, Columbus Children's Research Institute, The Ohio \\ State University, Columbus, OH, USA; ${ }^{2}$ Ohio State Biochemistry Program, The Ohio State University, \\ Columbus, OH, USA; ${ }^{3}$ Integrated Biomedical Science Graduate Program, The Ohio State University, \\ Columbus, OH, USA; ${ }^{4}$ Division of Medical Technology, School of Allied Medical Professions, The Ohio State \\ University, Columbus, OH, USA; ${ }^{5}$ Center for Injury Research and Policy, Columbus Children's Research \\ Institute, Columbus, OH, USA and ${ }^{6}$ Ohio State University Comprehensive Cancer Center, The Ohio State \\ University, Columbus, OH, USA
}

\begin{abstract}
Rhabdomyosarcoma is the most common pediatric soft-tissue sarcoma, which includes two major subtypes, alveolar and embryonal rhabdomyosarcoma. The mechanism of its oncogenesis is largely unknown. However, the oncogenic process of rhabdomyosarcoma involves multi-stages of signaling protein dysregulation characterized by prolonged activation of tyrosine and serine/threonine kinases. To better understand this protein dysregulation, we evaluated the phosphorylation profiles of multiple tyrosine and serine/threonine kinases to identify whether these protein kinases are activated in rhabdomyosarcoma. We applied immunohistochemistry with phospho-specific antibodies to examine phosphorylation levels of selected receptor and non-receptor tyrosine kinases, mammalian target of rapamycin (mTOR), p70S6K, and protein kinase $C(P K C)$ isozymes on alveolar and embryonal rhabdomyosarcoma tissue microarray slides. Our results showed that the phosphorylation levels of these kinases are elevated in some rhabdomyosarcoma tissues compared to normal tissues. Phosphorylation levels of receptor and non-receptor tyrosine kinases are elevated between 26 and $68 \%$ in alveolar rhabdomyosarcoma and between 24 and $71 \%$ in embryonal rhabdomyosarcoma, respectively, compared to normal tissues. In addition, phosphorylation levels of mTOR and its downstream targets, p70S6K, S6, and 4EBP1, are increased between 50 and $72 \%$ in both subtypes of rhabdomyosarcoma. Further, phosphorylation levels of $P K C \alpha, P K C \delta, P K C \theta$, and $P K C \zeta / \lambda$ are upregulated between 57 and $69 \%$ in alveolar rhabdomyosarcoma and between $\mathbf{4 3}$ and $\mathbf{7 2} \%$ in embryonal rhabdomyosarcoma. This is the first report to create a phosphorylation profile of tyrosine and serine/threonine kinases involved in the mTOR and PKC pathways of alveolar and embryonal rhabdomyosarcoma. These protein kinases may play roles in the development or tumor progression of rhabdomyosarcomas and thus may serve as novel targets for therapeutic intervention.
\end{abstract}

Modern Pathology (2007) 20, 936-946; doi:10.1038/modpathol.3800834; published online 22 June 2007

Keywords: $m T O R ; P K C$; rhabdomyosarcoma; tissue microarray; tyrosine kinases

Rhabdomyosarcoma is the most common soft tissue sarcoma in childhood. Approximately 350 cases are newly diagnosed each year in the United States and they account for $4 \%$ of all childhood malignancies. ${ }^{1}$ Most of these tumors arise in the head and neck region, genitourinary tract, and extremities. The two

Correspondence: Dr J Lin, PhD, Department of Pediatrics, WA5020, Center for Childhood Cancer, Columbus Children's Research Institute, The Ohio State University, Columbus, OH 43205, USA.

E-mail: lin.674@osu.edu

Received 20 February 2007; revised and accepted 23 May 2007; published online 22 June 2007 main histological subtypes of rhabdomyosarcoma are embryonal rhabdomyosarcoma, which comprises more than half of all rhabdomyosarcoma cases, and alveolar rhabdomyosarcoma, which is less common but more aggressive. These two subtypes arise at different primary body sites and have different age patterns. ${ }^{2}$ Clinically, the presentation of rhabdomyosarcoma is genetically heterogeneous. However, some genetic markers have been identified. Among them, approximately $75 \%$ of alveolar rhabdomyosarcoma are characterized by chromosomal translocation, most frequently, $\mathrm{t}(2 ; 13)(\mathrm{q} 35 ; \mathrm{q} 14)$ or the variant $\mathrm{t}(1 ; 13)(\mathrm{q} 36 ; \mathrm{q} 14))^{3}$ These translocations can disrupt either the PAX3 
or $P A X 7$ genes located on chromosomes 2 and 1 respectively, and the FKHR gene on chromosome 13, thereby generating the fusion protein $P A X 3-F K H R$ and $P A X 7-F K H R$ products. These fusion proteins function as chimeric transcription factors with increased transcription activity and have been shown to play a role in the dysregulation of the cell cycle in alveolar rhabdomyosarcoma cells. ${ }^{4,5}$

Rhabdomyosarcoma is believed to arise from the regulatory disruption of proliferation and differentiation of skeletal muscle progenitor cells, but the molecular etiology of the disease remains unclear. ${ }^{6}$ To shed light on this question, recent studies have compared the gene expression pattern in cancer cells versus surrounding normal tissue cells. ${ }^{7,8}$ The current methods for studying gene expression in cancer cells focuses on the mRNA level using cDNA microarray or on the protein level using proteomics technologies. ${ }^{9-12}$ Although these useful and powerful techniques do lead to the identification of differential expression of certain proteins at the mRNA or protein levels, they may not be able to detect the difference of proteins at the level beyond the post-translational modifications between cancer and normal cells.

Protein phosphorylation is one of the key posttranslational modifications to regulate protein function and appears to be most consistent with their biological activities. In normal cells, the phosphorylation state is under delicate control in that the protein kinases are usually phosphorylated transiently upon stimulation by growth factors or cytokines, whereas unstimulated cells have very low levels of phosphorylation of protein kinases. In cancer cells, however, some protein kinases show prolonged phosphorylation by upstream kinases or autophosphorylation and thus may be constitutively activated. This in turn leads to the constitutive activation of signal transduction pathways. Protein kinases, in particular many tyrosine kinases, are a major class of proto-oncogene products. ${ }^{13-16}$ Although tyrosine kinases account for less than $10 \%$ of all kinases, many of them are involved in growth signaling and cancer progression. ${ }^{17,18}$ These tyrosine kinases function in many diverse cellular processes such as growth, proliferation, mobility, survival, or tumor angiogenesis. The serine/threonine kinases represent another group of proteins important to cancer cell signaling. ${ }^{13}$ Serine/threonine kinases, such as mammalian target of rapamycin $(m T O R)$ and some protein kinase $\mathrm{C}(P K C)$ isozymes are frequently phosphorylated and become activated in multiple cancers. ${ }^{19,20}$ Tyrosine kinases, $m T O R$, and some $P K C$ isozymes are now regarded as excellent novel targets for cancer therapy. ${ }^{19,21-23}$ Given that protein kinases contribute as signaling mediators in tumorgenesis and tumor progression by activating downstream targets, and rhabdomyosarcomas are a substantial problem in pediatric oncology, there is a critical need to identify the phosphorylation profiles of signaling proteins in rhabdomyosarcomas.
We hypothesize that phosphorylation of some tyrosine and serine/threonine protein kinases is increased in rhabdomyosarcoma as compared to normal muscle cells. To address this question, we examined the phosphorylation of receptor and non-receptor tyrosine kinases and serine/threonine protein kinases involved in the mTOR and $P K C$ pathways of alveolar and embryonal rhabdomyosarcoma using immunohistochemistry staining on multiple rhabdomyosarcoma specimens that were fixed on paraffin-embedded tissue microarray slides. Phospho-specific antibodies that recognize phosphorylated proteins in those pathways were used in this study. This is a novel approach and should allow for the identification of new protein kinases not previously known to be involved in rhabdomyosarcoma oncogenesis. Our results show that the phosphorylation levels of many receptor and non-receptor tyrosine kinases and serine/threonine kinases in the $m T O R$ and $P K C$ pathways are frequently elevated in multiple rhabdomyosarcomas as compared to matched normal muscle tissues. These activated protein kinases may serve as novel therapeutic targets for treatment of patients with rhabdomyosarcoma.

\section{Materials and methods}

\section{Tissue Microarray Slides and Immunohistochemistry}

Human rhabdomyosarcoma tissue microarray slides were obtained from the Children's Oncology Group (COG). The embryonal rhabdomyosarcoma tissue microarray block contains 32 unique embryonal rhabdomyosarcoma cases with two types of control tissues: one control tissue is normal skeletal muscle (five cases) and the other is alveolar rhabdomyosarcoma (five cases). Each case averaged three tissue cores (range: 1-9 cores), which were each $1.0 \mathrm{~mm}$ in diameter and randomly distributed throughout the block. The alveolar rhabdomyosarcoma tissue microarray also contains 32 unique cases with identical block design as defined above. In addition to five normal skeletal muscle control cases, five embryonal rhabdomyosarcoma cases were included as controls. Rhabdomyosarcoma diagnoses were made via review by COG and STSC (Soft Tissue Sarcoma Committee), designated reviewers, using the International Classification of Rhabdomyosarcomas. ${ }^{24}$

These slides were baked at $60^{\circ} \mathrm{C}$ for $1 \mathrm{~h}$, deparaffinized in xylene three times, transferred through two changes of $100 \%$ ethanol, and then dehydrated with graded ethanol. Antigen retrieval was carried out by boiling the slides in a beaker filled with $10 \mathrm{mM}$ sodium citrate $(\mathrm{pH} 6.0)$ or $1 \mathrm{mM}$ EDTA $(\mathrm{pH}$ 8.0). Endogenous peroxidase activity was quenched through $10 \mathrm{~min}$ incubation in $3 \%$ hydrogen peroxide. After antigen retrieval, the slides were briefly rinsed with $0.1 \%$ Tween $/ 1 \times$ TBS (0.1\% TBST) two times and then washed three times for $10 \mathrm{~min}$ each 
at room temperature to remove non-specific background binding. The protein blocking was performed by incubating the specimens in 5\% normal goat serum or normal horse serum in $0.1 \%$ TBST for $1 \mathrm{~h}$. Primary antibody was applied at $4{ }^{\circ} \mathrm{C}$ overnight (1:50 dilution of rabbit monoclonal anti-human phospho-specific antibodies from Cell Signaling Technology, Beverly, MA, USA and Santa Cruz Biotechnology Inc., Santa Cruz, CA, USA) in $0.1 \%$ TBST with normal serum. The phospho-specific antibodies included phospho-EGFR (Y1068), phospho-PDGFR- $\beta$ (Y751), phospho-c-Met (Y1349), phospho-HER2/ErbB2 (Y1221/1222), phosphoVEGFR2 (Y1175), phospho-M-CSFR (Y723), phospho-FGFR (Y653/654), phospho-Bcr (Y177), phospho-c-Abl (Y245), phospho-c-Cbl (Y774), phospho-Src (Y416), phospho-JAK2 (Y1007/1008), phospho-mTOR (S2448), phospho-p70S6K (T389), phospho-S6 (S235/S236), phospho-4EBP1 (S65/ T70), phospho-PKC $\delta$ (T505), phospho-PKC $\theta$ (T538), phospho-PKC $\alpha$ (S657), phospho-PKC $\zeta / \lambda$ (T403/ T410) (Cell Signaling Technology), phospho-FAK (Y576/Y577), phospho-PYK2 (Y402), and phosphoShc (Y239/240) (Santa Cruz Biotechnology). After a series of rinses in TBS as described above, the bound antibody was subsequently detected using a VECTASTAIN ABC KIT from Vector Laboratories Inc. (Burlingame, CA, USA). The sections were then visualized with a 5-30 min incubation of 3-amino-9ethylcarbazole high-sensitivity substrate chromogen from DakoCytomation (Carpinteria, CA, USA). The slides were then counterstained with hematoxylin and mounted with CRYSTAL/MOUNT (Biomeda Corp., Foster City, CA, USA) for long- term preservation.

\section{Evaluation of Immunohistochemical Staining}

Immunohistochemical staining was scored under microscope by eye. The staining intensity was graded relatively based on the following scale: 0 , no staining; 1 , weak staining; 2 , moderate staining; and 3 , intense staining. ${ }^{25}$ Most or all of the tumor tissues showed staining in greater than $50 \%$ of the area. For specimens that were uninterpretable or in which most of the cancer tissues were missing, a score of not applicable (N/A) was given. Scoring of the tissue microarray was completed by at least two independent observers (LC and FH or KA and FH). Discrepant scores between the two observers were rescored to arrive at a single final score. For a tumor specimen to be considered positive, it had to have a score of 2 or greater from both observers.

\section{Statistical Analysis}

SAS software (version 9.1, SAS Institute Inc., Cary, NC, USA) was used for all statistical analysis. Fisher's exact probability test was used to investigate the correlation of phospho-protein kinase expression with the following potential predictive variables: gender, age, stage at diagnosis, and tumor primary site. When Fisher's exact test is significant in comparison among multiple groups, we then categorized data into $2 \times 2$ tables and used Fisher's exact test to make significant conclusions. Spearman's model was used to investigate the association between phosphorylation levels of different protein kinases. In all analyses, a two-sided $P$-value of $<0.05$ was considered statistically significant.

\section{Results}

\section{Increased Phosphorylation of Receptor Tyrosine Kinases in Rhabdomyosarcomas}

More than 30 receptor tyrosine kinases have been reported to play a role in oncogenic transformation. ${ }^{13}$ Among them, EGFR, Her2/ErbB2, and $c$-Met are reported to be constitutively activated, promoting the activation of downstream signaling processes that induce cell growth, cell survival, and invasion of cancer cells. ${ }^{26}$ These kinases become activated when they are phosphorylated by upstream kinases or through autophosphorylation. To further understand the phosphorylation profile of receptor and non-receptor tyrosine kinases in alveolar and embryonal rhabdomyosarcoma, we examined specimens that were fixed on paraffinembedded tissue microarray slides (Table 1). The patient ages of these cases are between 0 and 19 with mean 9.1 in alveolar rhabdomyosarcoma and 5.8 in embryonal rhabdomyosarcoma, and most of the cases are in stage III (Table 1). Seven receptor tyrosine kinases, which include $c$-Met, PDGFR- $\beta$,

Table 1 Patient clinical characteristics

\begin{tabular}{lcc}
\hline Characteristic & \multicolumn{2}{c}{ Number of cases } \\
\cline { 2 - 3 } & ARMS $(\mathrm{n}=32)$ & ERMS $(\mathrm{n}=32)$ \\
\hline Gender & & \\
Male & 13 & 24 \\
Female & 19 & \\
& & \\
Age (years) & 11 & 18 \\
$0-4$ & 14 & 4 \\
$5-14$ & 7 & 5.8 \\
15-19 & 9.1 & \\
Mean & & 7 \\
& & 4 \\
Stage & 5 & 7 \\
I & 5 & \\
II & 15 & 10 \\
III & 7 & 3 \\
IV & & 9 \\
Primary site & 9 & \\
Head and neck & 13 & \\
Extremity & 3 & \\
Genitourinary & & \\
Others & & \\
& &
\end{tabular}


EGFR, M-CSFR, Her2/ErbB2, VEGFR-2/FIK-1, and $F G F R$, were selected. These receptor tyrosine kinases fall into six subfamilies. The phosphorylation levels of these receptor tyrosine kinases are elevated between 26 and 68\% in alveolar rhabdomyosarcoma and between 24 and $61 \%$ in embryonal rhabdomyosarcoma, respectively (Table 2). In all, $45 \%$ or greater of both alveolar and embryonal rhabdomyosarcoma cases demonstrated positive staining for tyrosine phosphorylation of $C$-Met, $P D G F R-\beta$, and $M-C S F R$ suggesting that these pathways may play a role in the oncogenesis of both types of rhabdomyosarcomas. The increased level of tyrosine phosphorylation of EGFR is around $50 \%$ in alveolar rhabdomyosarcoma indicating the EGFR pathway may play a role in cancer progression in this type of rhabdomyosarcoma. Furthermore, the increased levels of tyrosine phosphorylation of Her2/ErbB2, FGFR and VEGFR-2/FIK-1 are between 24 and $44 \%$, suggesting that these pathways might also contribute to oncogenesis in rhabdomyosarco-

Table 2 Elevated phosphorylation of kinases and their downstream proteins in ARMS and ERMS patients ${ }^{\mathrm{a}}$

Positive (\%)

ARMS $(\mathrm{n}=32) \quad$ ERMS $(\mathrm{n}=32)$

Receptor tyrosine kinase
p-c-Met (Y1349)
p-PDGFR- $\beta$ (Y751)
p-EGFR (Y1068)
p-M-CSFR (Y723)
p-HER2/ErbB2 (Y1221/1222)
p-VEGFR2 (Y1175)
p-FGFR (Y653/654)

$\begin{array}{ll}68 & 57 \\ 64 & 57 \\ 50 & 31 \\ 45 & 61 \\ 44 & 24 \\ 32 & 52 \\ 26 & 38\end{array}$

Non-receptor tyrosine kinase p-JAK2 (Y1007/1008)

p-PYK2 (Y402)

p-Src (Y416)

p-c-Abl (Y245)

p-c-Cbl (Y774)

p-Bcr (Y177)

p-FAK (Y576/577)

p-Shc (Y239/240)

$\begin{array}{ll}65 & 70 \\ 30 & 48 \\ 26 & 43 \\ 50 & 48 \\ 41 & 34 \\ 57 & 71 \\ 63 & 64 \\ 64 & 71\end{array}$

mTOR pathway

p-mTOR (S2448)

p-p70S6K (T389)

p-S6 (S235/S236)

p-4EBP1 (S65/T70)

$\begin{array}{ll}60 & 68 \\ 65 & 72 \\ 50 & 59 \\ 62 & 71\end{array}$

PKC pathway

$\mathrm{p}-\mathrm{PKC} \alpha(\mathrm{T} 657)$

$\mathrm{p}-\mathrm{PKC} \delta$ (T505)

p-PKC $\theta$ (T538)

$\mathrm{p}-\mathrm{PKC} \zeta / \lambda(\mathrm{T} 403 / \mathrm{T} 410)$

$\begin{array}{ll}68 & 43 \\ 69 & 64 \\ 65 & 69 \\ 57 & 72\end{array}$

${ }^{\mathrm{a}}$ The immunostaining intensity was scored under microscope on the following scale: 0 , no staining; 1 , weak staining; 2 , moderate staining; and 3 , intense staining. Scoring of the tissue microarray was completed by at least two independent researchers. Scores of normal tissues are 0 or 1 . Scores of 2 and 3 of IHC staining in ARMS and ERMS tissues were considered as positive. \% positive: percentage of numbers of positive divided by the total number of specimens. mas. Representative positive immunohistochemistry staining for phospho-EGFR and phospho-c-Met in rhabdomyosarcoma patient tissues are shown in Figure 1. The immunohistochemistry staining of corresponding phospho-protein in normal tissues were negative (Figure 1). The relationship between the expression of different phospho-receptor tyrosine kinases and patient gender, age, tumor stage, and primary sites was analyzed. Neither patient gender nor tumor stage was found to be statistically significantly correlated with the expression of phospho-receptor tyrosine kinases in both alveolar and embryonal rhabdomyosarcoma cases. Interestingly, the expression of phospho-M-CSFR was found to be significantly correlated with patient age in alveolar rhabdomyosarcoma patients $(P=0.0062)$. The alveolar rhabdomyosarcoma cases that showed higher phosphorylation of M-CSFR (immunohistochemistry staining score of 2 or 3 ) are younger compared to those cases with lower phospho-MCSFR levels (immunohistochemistry staining score of 0 or 1, Table 3 ). A total of 7 out of 10 alveolar rhabdomyosarcoma patients who have higher $p-M$ CSFR expression are in the age group 0-4 (data not shown). The expression of phospho-FGFR was found to be significantly correlated with patient age in embryonal rhabdomyosarcoma patients $(P=0.0266)$. The embryonal rhabdomyosarcoma cases with higher phosphorylation of FGFR are older compared to those cases with lower phospho-FGFR levels (Table 3). A total of 8 out of 11 embryonal rhabdomyosarcoma patients who displayed higher phospho-FGFR expression are older than age 4 (data not shown). Furthermore, the expression of phospho-PDGFR was found to be significantly correlated with tumor primary sites in embryonal rhabdomyosarcoma $(P=0.0183)$. The embryonal rhabdomyosarcoma cases that showed higher phospho-PDGFR levels arise more frequently from head and neck region as well as extremity, whereas those express lower phospho-PDGFR arise more frequently from other sites (Table 3 ). Statistical analysis also showed that the expression of some phospho-receptor tyrosine kinases is correlated with each other. For example, the expression of phospho$c$-Met and phospho-EGFR is significantly correlated $(P<0.0001)$ in both alveolar and embryonal rhabdomyosarcoma patients (Table 4). More correlations between the expressions of phosphorylated receptor tyrosine kinases are shown in Table 4.

\section{Increased Phosphorylation Levels of Non-Receptor Tyrosine Kinases in Rhabdomyosarcomas}

We also tested tyrosine phosphorylation levels of eight non-receptor kinases in alveolar and embryonal rhabdomyosarcoma respectively, which include JAK2, PYK2, Src, $C$-Abl, $C$-Cbl, Bcr, FAK, and Shc. Among them, Src activation has been shown to be correlated with cancer progression. ${ }^{15}$ Our results 


\section{p-EGFR (Y1068)}

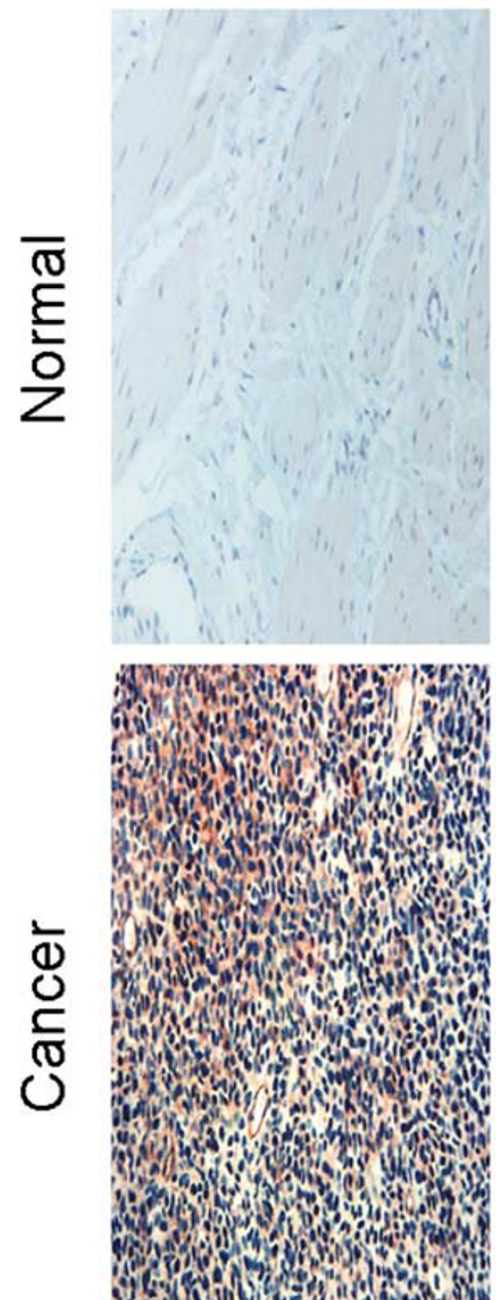

ARMS
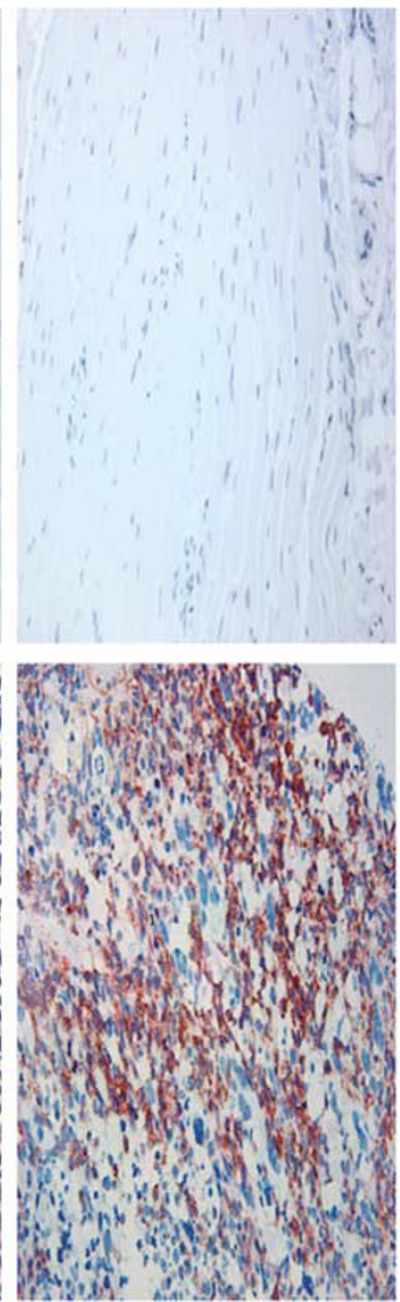

ERMS
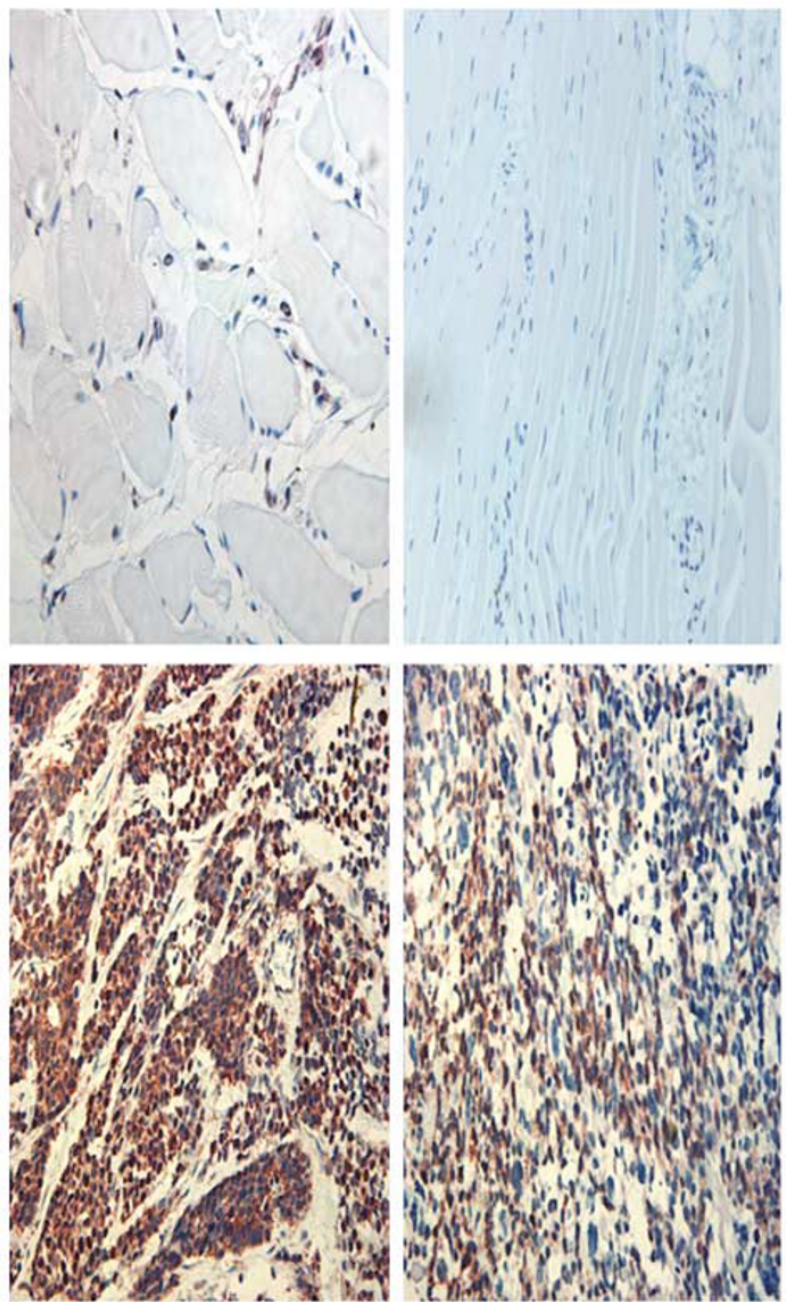

ARMS

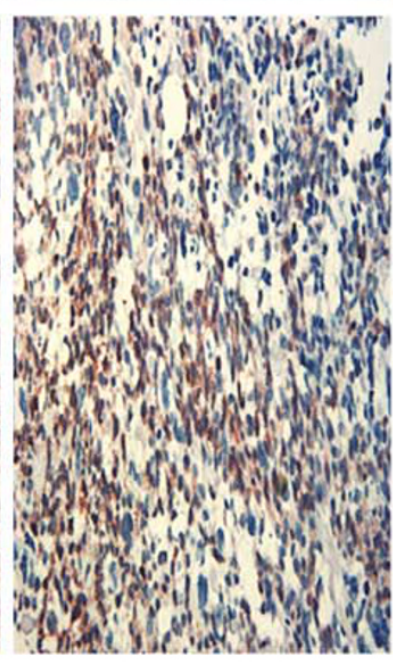

ERMS

Figure 1 Elevated phosphorylation levels of representative receptor tyrosine kinases, EGFR and $c$-Met, in rhabdomyosarcoma. Immunohistochemistry staining of alveolar and embryonal rhabdomyosarcoma tissue microarrays that contain both normal and cancer tissues were performed using phospho-EGFR (Tyr1068) antibody and phospho-c-Met (Tyr1349) antibody.

Table 3 Significant correlation between immunohistochemical expression of tyrosine kinases and clinical characteristics in ARMS and ERMS

Age

P-value

\begin{tabular}{lccc}
\cline { 2 - 3 } & High $^{\mathrm{a}}$ & Low $^{\mathrm{a}}$ & \\
\hline p-M-CSFR (ARMS) & $0-4$ years & 5-19 years & 0.0062 \\
p-FGFR (ERMS) & $5-19$ years & $0-4$ years
\end{tabular}

p-FGFR (ERMS)

p-PDGFR (ERMS)

p-Src (ERMS)
Head and neck extremity Head and neck extremity

Primary sites

${ }^{\mathrm{a}}$ High, IHC-staining score of 2 and 3; low, IHC-staining score of 0 and 1.

showed that the phosphorylation levels of these eight non-receptor tyrosine kinases are elevated between 26 and $65 \%$ in alveolar rhabdomyosarcoma and between 34 and $71 \%$ in embryonal rhabdomyosarcoma (Table 2). The increased levels of tyrosine phosphorylation of JAK2, $c-A b l, B c r, F A K$, and Shc 
are over or around $50 \%$ in both alveolar and embryonal rhabdomyosarcoma suggesting that these five pathways may play important roles in the oncogenesis of both types of rhabdomyosarcomas. Furthermore, the increased levels of tyrosine phosphorylation of $\mathrm{PYK} 2, \mathrm{Src}$, and $\mathrm{C}$-Cbl are between 26 and $48 \%$ indicating these pathways might also be involved in the oncogenesis of both alveolar and embryonal rhabdomyosarcoma. Representative rhabdomyosarcoma patient specimens staining positive for phospho-c-Cbl and phospho-Src are shown in Figure 2. No significant correlation was found between the expression of phosphonon-receptor tyrosine kinases and patient gender, age or tumor stage in both alveolar and embryonal rhabdomyosarcoma. In embryonal rhabdomyosarcoma, the phospho-Src level is found to be significantly correlated with tumor primary sites $(P=0.0189)$. The embryonal rhabdomyosarcoma cases that showed higher phosphorylation of SrC arise more frequently from head and neck region and extremity (Table 3). Statistical analysis also showed that the expression of some phosphonon-receptor tyrosine kinase is correlated with each other. As an example, the expression of phosphoSrc and phospho-C-Abl is statistically correlated in alveolar rhabdomyosarcoma $(P<0.0001)$ and embryonal rhabdomyosarcoma $(P=0.0194)$ patients (Table 5). Correlations between the expressions of phosphorylated non-receptor tyrosine kinases are detailed in Table 5.

\section{The Phosphorylation Levels of Protein Kinases in mTOR Pathways are Elevated in Rhabdomyosarcomas}

The serine/threonine protein kinases, mTOR and p70S6K, are among the most important downstream effectors in oncogenic receptor tyrosine kinase signaling. ${ }^{27}$ We examined the phosphorylation levels of mTOR and p70S6K as well as their downstream-targeted proteins, $S 6$ and $4 E B P 1$ that function in protein translation. Our results showed that phosphorylation levels of mTOR are elevated $60 \%$ in alveolar rhabdomyosarcoma and $68 \%$ in embryonal rhabdomyosarcoma. Phosphorylation of p70S6K and $S 6$ are also increased between 50 and $72 \%$ in both types of rhabdomyosarcoma (Table 2). Positive immunohistochemistry staining for phospho-p70S6K in rhabdomyosarcoma patient tissues compared to normal tissues are shown in Figure 3. No evidence of significant correlation was found between any clinicopathological features and the expression of phosphorylated mTOR or p70S6K in both alveolar and embryonal rhabdomyosarcoma patients. Statistical analysis showed that the expression of phospho-mTOR and phospho-p70S6K is significantly correlated in alveolar rhabdomyosarcoma $(P<0.0001)$ and embryonal rhabdomyosarcoma $(P=0.0179)$ patients (data not shown). 


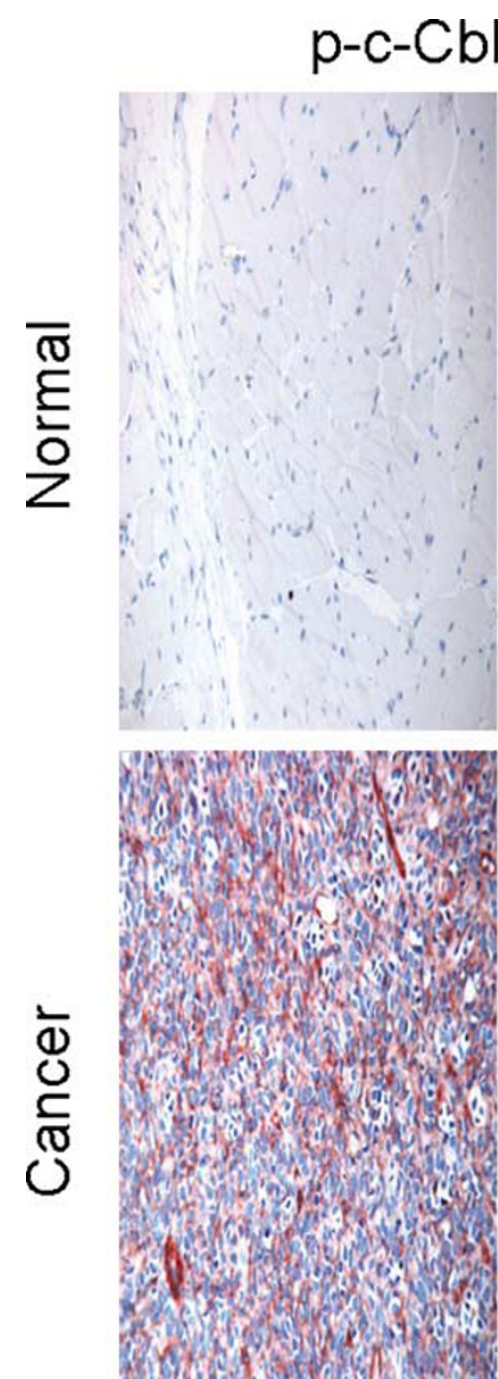

ARMS
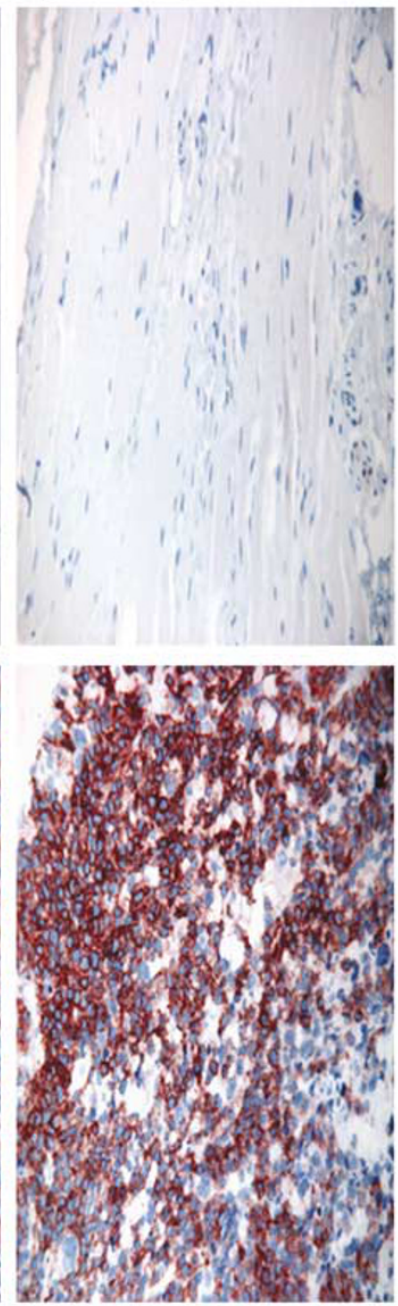

ERMS
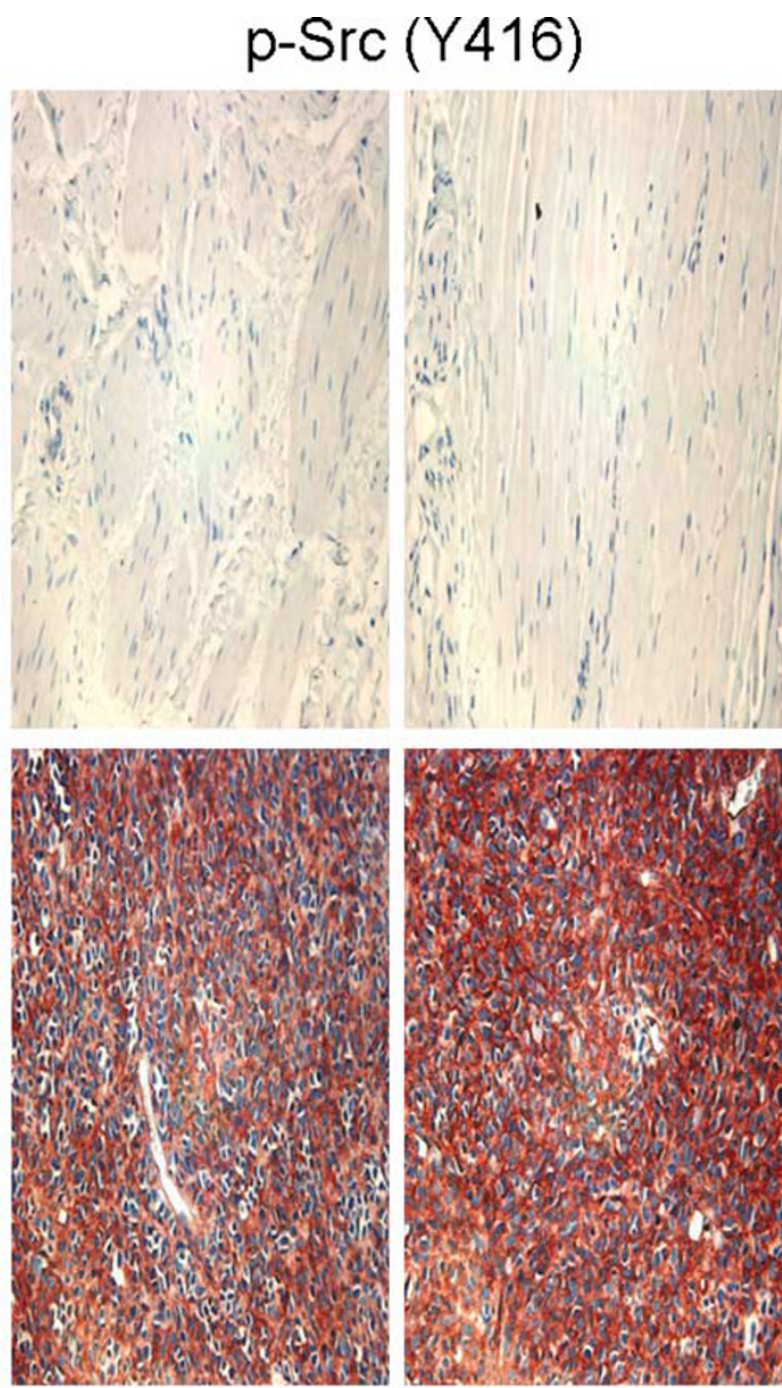

ARMS

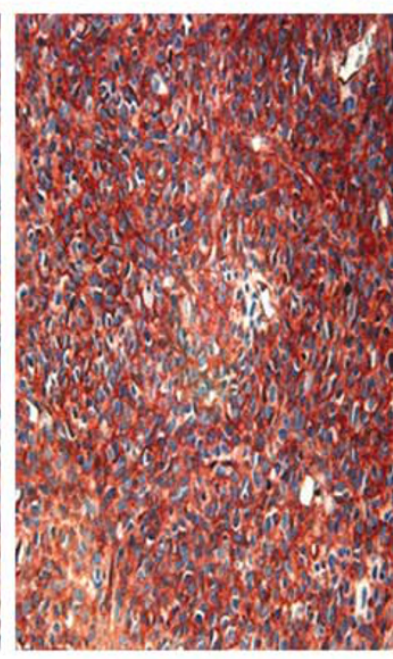

ERMS

Figure 2 Elevated phosphorylation levels of representative non-receptor tyrosine kinases, $c$-Cbl and Src, in rhabdomyosarcoma. Immunohistochemistry staining of alveolar and embryonal rhabdomyosarcoma tissue microarrays that contain both normal and cancer tissues were performed using phospho-c-Cbl (Tyr774) and phospho-Src (Tyr416) antibodies.

\section{Elevated Phosphorylation Levels of Protein Kinase C Isozymes in Rhabdomyosarcomas}

The $P K C$ enzyme family contains 12 different isozymes, many of which contribute to the regulation of diverse cellular response including proliferation, differentiation, and apoptosis. ${ }^{28}$ The phosphorylation state of an isozyme is a crucial factor in determining activity. ${ }^{29,30}$ We examined four $P K C$ isozymes which are serine/threonine kinases. Phosphorylation levels of $P K C \alpha, P K C \delta, P K C \theta$, and $P K C \zeta / \lambda$ are significantly upregulated between 57 and $69 \%$ in alveolar rhabdomyosarcoma, between 43 and $72 \%$ in embryonal rhabdomyosarcoma (Table 2). Positive immunohistochemistry staining for phospho-PKC $\delta$ of rhabdomyosarcoma patient tissues are shown in Figure 3. No significant correlation exists between any clinicopathological features with the expression of phospho-PKC isozymes in both alveolar and embryonal rhabdomyosarcoma patients. The correlation between the expression of phosphorylated $P K C \alpha$ and alveolar rhabdomyosarcoma patient age is close to significance $(P=0.0757)$. In alveolar rhabdomyosarcoma patients, the expression of phospho-PKC $\theta$ and phospho-PKC $\delta$ is found to be significantly correlated $(P=0.0078$, data not shown). In embryonal rhabdomyosarcoma patients, the expression of phospho$P K C \alpha$ and phospho-PKC $\delta$ is significantly correlated $(P=0.0006$, data not shown $)$.

\section{Discussion}

In this study, we identified for the first time, the phosphorylation levels of multiple tyrosine kinases 


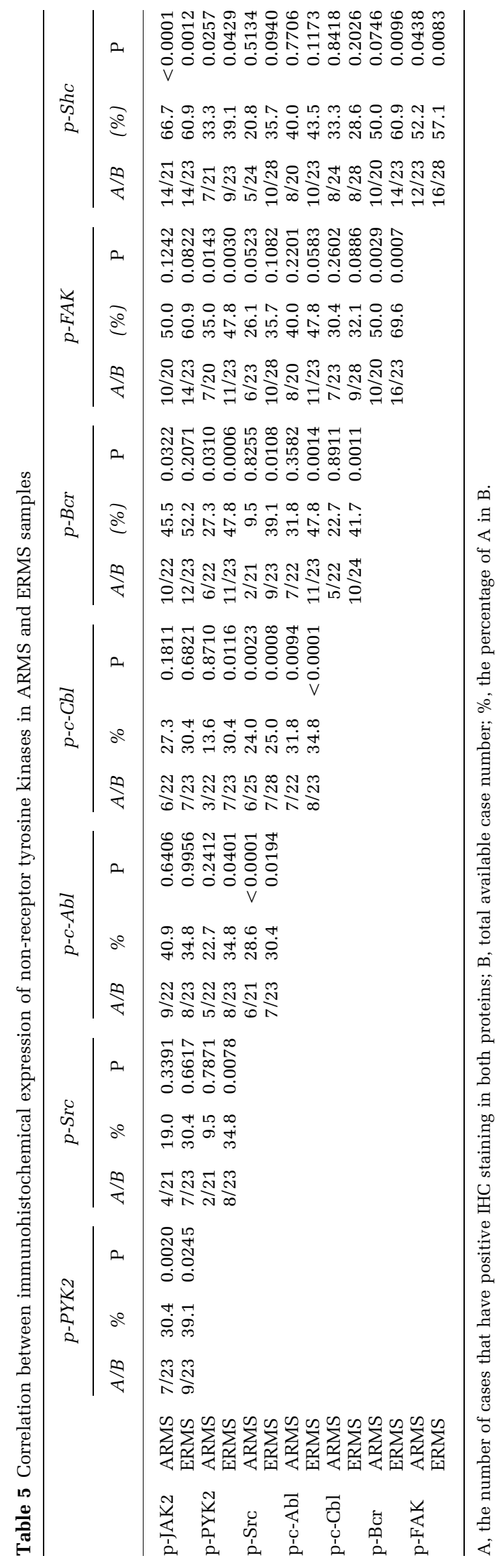

as well as serine/threonine kinases in the mTOR and $P K C$ pathways that are elevated in alveolar and embryonal rhabdomyosarcoma tissues. We showed that increased phosphorylation levels of seven selected receptor tyrosine kinases are between 24 and $68 \%$ in alveolar and embryonal rhabdomyosarcoma. Our results also showed that the phosphorylation levels of these eight non-receptor tyrosine kinases are elevated between 26 and 71\% in alveolar and embryonal rhabdomyosarcoma. Further, we showed that increased phosphorylation levels of serine/threonine kinases in the mTOR and $P K C$ pathways are between 43 and $72 \%$ in alveolar and embryonal rhabdomyosarcoma. We have also been able to obtain phosphorylation profiles of these selected tyrosine kinases and serine/threonine kinases in the mTOR and PKC pathways from rhabdomyosarcoma patient tissues. These protein kinases have been shown to contribute to cell proliferation and cell survival ${ }^{31-42}$ and most of them are likely to play crucial roles in tumor development and progression of rhabdomyosarcomas. Therefore, selective small molecular inhibitors that block these activated protein kinases may be useful therapeutic reagents.

Statistical analysis of these data showed that the elevated levels of phosphorylation of multiple protein kinases are correlated in rhabdomyosarcoma patient samples. The elevated phosphorylation of EGFR is significantly correlated with $C$-Met, PDGFR, Her2/ErbB2, FGFR, and VEGFR, respectively $(P$-values $<0.05)$ but not with $M$-CSFR, whereas the elevated expression of phospho-C-Met is correlated with phospho-EGFR, phospho-FGFR, and phospho-VEGFR but not with phospho-MCSFR, phospho-PDGFR, and phospho-Her2/ErbB2 in alveolar rhabdomyosarcoma (Table 4). The elevated phosphorylation of VEGFR is correlated with $c$-Met, EGFR, M-CSFR, and PDGFR, respectively $(P$-values $<0.05)$ and the elevated phosphorylation of EGFR is correlated with $c$-Met, PDGFR, and M-CSFR in alveolar rhabdomyosarcoma (Table 4). These results suggest that in these 32 alveolar rhabdomyosarcoma, there are at least two or more receptor tyrosine kinases whose phosphorylation is elevated in the same patient. In embryonal rhabdomyosarcoma, for example, the expression of phospho-EGFR is significantly correlated with phospho-c-Met, phospho-PDGFR, phospho-Her2/ErbB2, and phosphoFGFR, but not with phospho-VEGFR or phospho$M$-CSFR. The elevated expression of phospho-C-Met is correlated with phospho-EGFR, phospho-FGFR, phospho-M-CSFR, phospho-PDGFR, and phosphoVEGFR but not with phospho-Her2/ErbB2 (Table 4). These results also suggest that in these 32 embryonal rhabdomyosarcoma, there are at least two or more receptor tyrosine kinases whose phosphorylation is elevated in the same patient. Elevated expression of two or more receptor tyrosine kinases is presented in only alveolar or embryonal rhabdomyosarcoma but not in both subtypes. For example, in Table 3, 

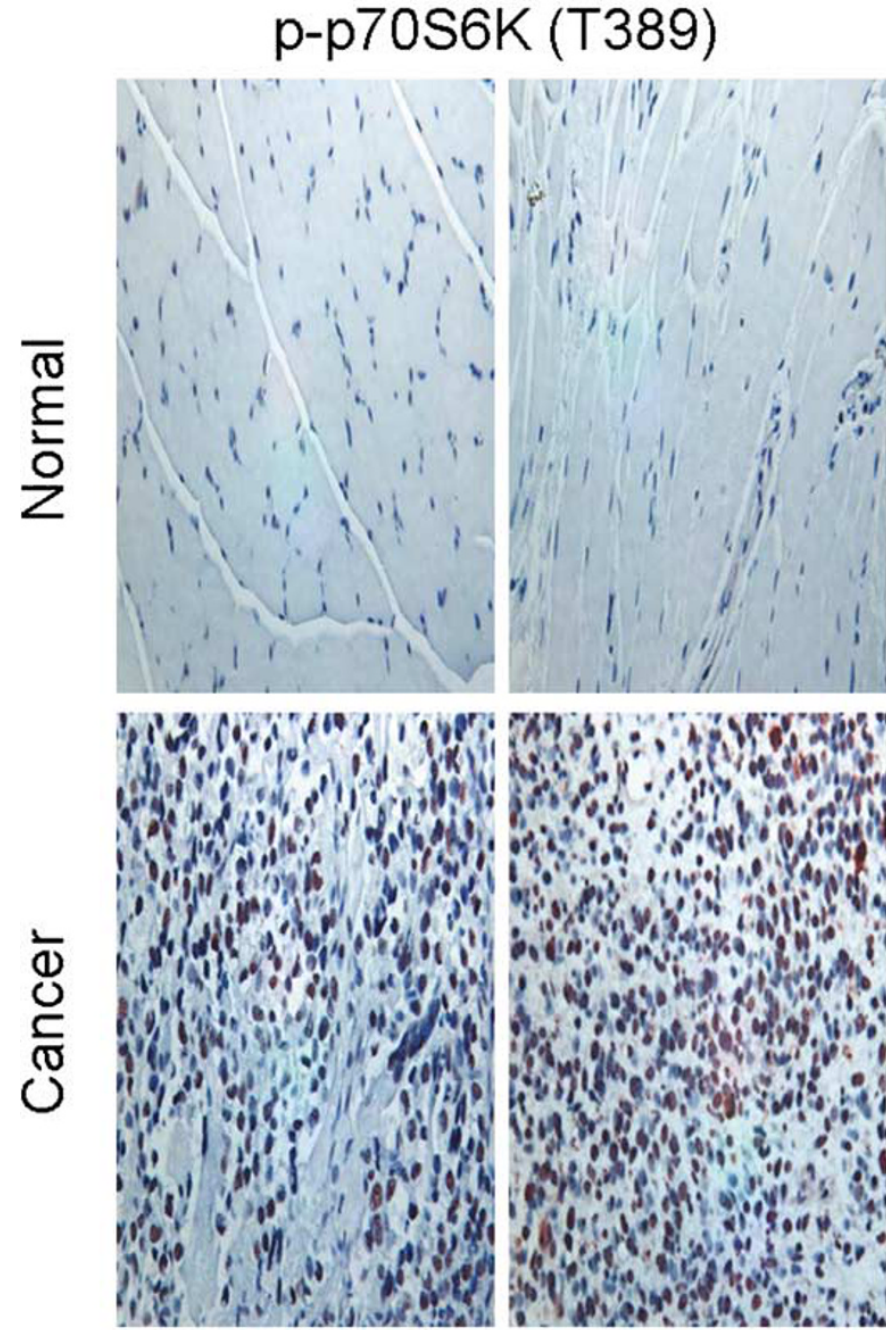

ARMS

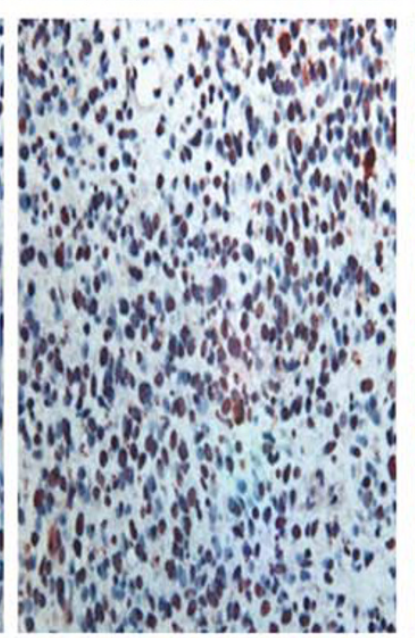

ERMS
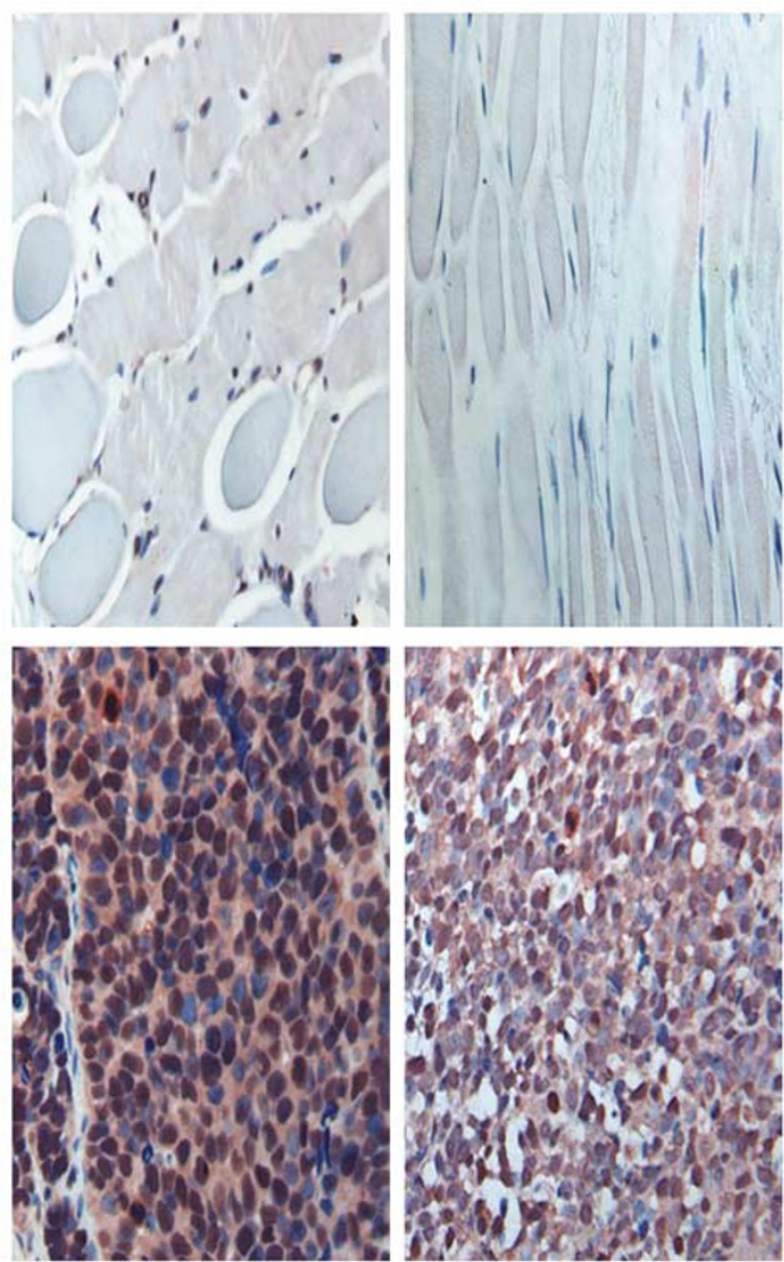

ARMS

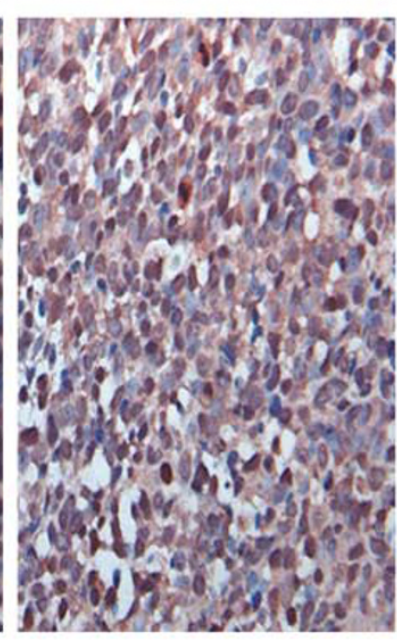

ERMS

Figure 3 Elevated phosphorylation levels of representative serine/threonine kinases, $p 70 S 6 K$ and $P K C \delta$, in rhabdomyosarcoma. Immunohistochemistry staining of alveolar and embryonal rhabdomyosarcoma tissue microarrays that contain both normal and cancer tissues were performed using phospho-p70S6K (Thr389) and phospho-PKC $\delta$ (Thr505) antibodies.

the elevated expression of phospho-PDGFR is significantly correlated with phospho-C-Met only in embryonal rhabdomyosarcoma $(P=0.0001)$ but not in alveolar rhabdomyosarcoma $(P=0.0659)$ and the elevated expression of phospho-VEGFR is significantly correlated with phospho-EGFR only in alveolar rhabdomyosarcoma $(P=0.0016)$ but not in embryonal rhabdomyosarcoma $(P=0.3441)$. These results suggest that co-activation of these two receptor tyrosine kinases through their downstream signaling pathways may be important for the cooperation in tumor development or progression in either alveolar rhabdomyosarcoma or embryonal rhabdomyosarcoma but may not be important in another subtype. In addition, the elevated expression of several receptor tyrosine kinases is not associated with each other at all in both alveolar rhabdomyosarcoma and embryonal rhabdomyosar- coma. The elevated expression of phospho-M-CSFR and phospho-EGFR $(P=0.1276$ in alveolar rhabdomyosarcoma and $P=0.1150$ in embryonal rhabdomyosarcoma) and phospho-FGFR and phosphoVEGFR $(P=0.2168$ in alveolar rhabdomyosarcoma and $P=0.0895$ in embryonal rhabdomyosarcoma) are not associated with each other in both alveolar and embryonal rhabdomyosarcoma (Table 4). These results suggest that co-activation of these two receptor tyrosine kinases and their downstream signaling pathways may not be relevant for the cooperation in tumor development or progression in either alveolar rhabdomyosarcoma or embryonal rhabdomyosarcoma.

Among the non-receptor tyrosine kinases, some of their increased phosphorylation is also correlated in both subtypes of rhabdomyosarcoma. For example, the expression of PYK2 is significantly correlated 
with JAK2, Bcr, FAK, and Shc in both alveolar and embryonal rhabdomyosarcoma $(P$-values $<0.05$, Table 5). Furthermore, oncogene products that are activated in cancer such as $S r c, c-A b l, B c r$, and $c$-Cbl are associated with each other in some cases of alveolar and embryonal rhabdomyosarcoma. For example, significant correlations were observed between the expression of phospho-Src and phospho-C-Abl, phospho-Src and phospho-c-Cbl, phospho-c-Abl and phospho-c-Cbl, in both alveolar and embryonal rhabdomyosarcoma (Table 5). These findings suggest that the concurrent elevated phosphorylation and activation of at least two of these non-receptor tyrosine kinases that may function as oncogene products may contribute to the signaling pathway dysregulation and tumor formation or progression in rhabdomyosarcoma. The elevated phosphorylation of certain non-receptor tyrosine kinases are only associated with each other in one subtype of rhabdomyosarcoma. For instance, the significant association of increased levels of phosphorylation of $B c r$ and $C-A b l$ only exists in embryonal rhabdomyosarcoma $(P=0.0014)$ but not in alveolar rhabdomyosarcoma $(P=0.3582$, Table 5$)$. In addition, the significant association of increased levels of phosphorylation of $B C r$ and $S r c$ is only observed in embryonal rhabdomyosarcoma $(P=0.0108)$ but not in alveolar rhabdomyosarcoma $(P=0.8255$, Table 5), suggesting that the co-activation of $B c r$ and $C-A b l$, or BCr and Src might play an important role in tumorigenesis or development of embryonal rhabdomyosarcoma. Further, the elevated phosphorylation of several receptor tyrosine kinases is not associated with each other at all in both alveolar rhabdomyosarcoma and embryonal rhabdomyosarcoma. For example in Table 5, the elevated phosphorylation of $J A K 2$ and $S r c$ or $C-A b l$ and $F A K$ is not significantly associated with each other in both alveolar and embryonal rhabdomyosarcoma (Table 5). This suggests that co-activation of these non-receptor tyrosine kinases and their downstream signaling pathways might not promote oncogenesis in alveolar and embryonal rhabdomyosarcoma. Instead, co-activation of Src, $c-A b l, F A K$, or $J A K 2$ with others, such as, $S r c$ with $c$-Abl, JAK2 with $S h c$, or FAK with Bcr (Table 5) might cooperate more efficiently in promoting tumor development or progression in both alveolar and embryonal rhabdomyosarcoma.

Findings from this pilot study may have potential clinical utility and may be used to optimize the molecular therapeutic targets for individualized cancer interventions. To the best of our knowledge, this is the first report to profile the phosphorylation levels of a variety of receptor tyrosine, non-receptor tyrosine, and serine/threonine kinases in the mTOR and PKC pathways in rhabdomyosarcomas. This report may provide a basis for future studies using small molecule compounds and other intervention methods that target these activated protein kinases in rhabdomyosarcomas.

\section{Acknowledgement}

This work was supported in part by the Elsa U Pardee Foundation and a start-up fund from the Department of Pediatrics at the Ohio State University.

\section{References}

1 Ries L, Smith M, Gurney J, et al. Cancer incidence and survival among children and adolescents: United States SEER Program 1975-1995. National Cancer Institute, SEER Program. NIH Pub. No. 99-4649 Bethesda, MD, 1999.

2 McDowell H. Update on childhood rhabdomyosarcoma. Arch Dis Child 2003;88:354-357.

3 Sorensen PHB, Lynch JC, Qualman SJ, et al. PAX3FKHR and PAX7-FKHR gene fusions are prognostic indicators in alveolar rhabdomyosarcoma: a report from the Children's Oncology Group. J Clin Oncol 2002;20:2672-2679.

4 Davis R, D’Cruz C, Lovell M, et al. Fusion of PAX7 to FKHR by the variant $\mathrm{t}(1 ; 13)(\mathrm{p} 36 ; \mathrm{q} 14)$ translocation in alveolar rhabdomyosarcoma. Cancer Res 1994;54: 2869-2872.

5 Shapiro DN, Sublett JE, Li B, et al. Fusion of PAX3 to a member of the forkhead family of transcription factors in human alveolar rhabdomyosarcoma. Cancer Res 1993;53:5108-5112.

6 Merlino G, Helman LJ. Rhabdomyosarcoma-working out the pathways. Oncogene 1999;18:5340-5348.

7 Bortoluzzi S, Bisognin A, Romualdi C, et al. Novel genes, possibly relevant for molecular diagnosis or therapy of human rhabdomyosarcoma, detected by genomic expression profiling. Gene 2005;348:65-71.

8 Khan J, Wei J, Ringner M, et al. Classification and diagnostic prediction of cancers using gene expression profiling and artificial neural networks. Nat Med 2001;7:673.

9 DeRisi J, Penland L, Brown P, et al. Use of a cDNA microarray to analyse gene expression patterns in human cancer. Nat Genet 1996;14:457-460.

10 Khan J, Simon R, Bittner M, et al. Gene expression profiling of alveolar rhabdomyosarcoma with cDNA microarrays. Cancer Res 1998;58:5009-5013.

11 Issaq H, Veenstra T, Conrads T, et al. The SELDI-TOF MS approach to proteomics: protein profiling and biomarker identification. Biochem Biophys Res Commun 2002;292:587.

12 Sreekumar A, Nyati MK, Varambally S, et al. Profiling of cancer cells using protein microarrays: discovery of novel radiation-regulated proteins. Cancer Res 2001;61:7585-7593.

13 Blume-Jensen P, Hunter T. Oncogenic kinase signalling. Nature 2001;411:355-365.

14 Paul M, Mukhopadhyay A. Tyrosine kinase-role and significance in cancer. Int J Med Sci 2004;1: 101-115.

15 Irby R, Yeatman T. Role of Src expression and activation in human cancer. Oncogene 2000;19:56365642.

16 Vlahovic G, Crawford J. Activation of tyrosine kinases in cancer. Oncologist 2003;8:531-538.

17 Kameda T, Yasui W, Yoshida K, et al. Expression of ERBB2 in human gastric carcinomas: relationship 
between p185ERBB2 expression and the gene amplification. Cancer Res 1990;50:8002-8009.

18 Kuniyasu H, Yasui W, Kitadai Y, et al. Frequent amplification of the c-met gene in scirrhous type stomach cancer. Biochem Biophys Res Commun 1992; 189:227-232.

19 Altomare DA, Wang HQ, Skele KL, et al. AKT and mTOR phosphorylation is frequently detected in ovarian cancer and can be targeted to disrupt ovarian tumor cell growth. Oncogene 2004;23:5853-5857.

20 Lahn M, Kohler G, Sundell K, et al. Protein kinase C alpha expression in breast and ovarian cancer. Oncology 2004;67:1-10.

21 Krause D, Van Etten R. Tyrosine kinases as targets for cancer therapy. N Engl J Med 2005;353:172-187.

22 Longati P, Comoglio P, Bardelli A. Receptor tyrosine kinases as therapeutic targets: the model of the MET oncogene. Curr Drug Targets 2001;2:41-55.

23 Alkan S, Huang Q, Ergin M, et al. Survival role of protein kinase $\mathrm{C}$ (PKC) in chronic lymphocytic leukemia and determination of isoform expression pattern and genes altered by PKC inhibition. Am J Hematol 2005;79:97-106.

24 Qualman S, Coffin C, Newton W, et al. Intergroup rhabdomyosarcoma study: update for pathologists. Pediatr Dev Pathol 1998;1:550.

25 Dolled-Filhart M, Camp R, Kowalski D, et al. Tissue microarray analysis of signal transducers and activators of transcription 3 (Stat3) and phospho-Stat3 (Tyr705) in node-negative breast cancer shows nuclear localization is associated with a better prognosis. Clin Cancer Res 2003;9:594-600.

26 Harari D, Yarden Y. Molecular mechanisms underlying ErbB2/HER2 action in breast cancer. Oncogene 2000;19:6102-6114.

27 Vivanco I, Sawyers CL. The phosphatidylinositol 3kinase-AKT pathway in human cancer. Nat Rev Cancer 2002;2:489-501.

28 Dempsey E, Newton A, Mochly-Rosen D, et al. Protein kinase $\mathrm{C}$ isozymes and the regulation of diverse cell responses. Am J Physiol Lung Cell Mol Physiol 2000;279:L429-L438.

29 Dutil E, Toker A, Newton A. Regulation of conventional protein kinase $\mathrm{C}$ isozymes by phosphoinositidedependent kinase 1 (PDK-1). Curr Biol 1998;8: 1366-1375.

30 Le Good J, Ziegler W, Parekh D, et al. Protein kinase C isotypes controlled by phosphoinositide 3-kinase through the protein kinase PDK1. Science 1998;281:2042-2045.
31 Brasher B, Van Etten R. c-Abl has high intrinsic tyrosine kinase activity that is stimulated by mutation of the Src homology 3 domain and by autophosphorylation at two distinct regulatory tyrosines. J Biol Chem 2000;275:35631-35637.

32 Claesson-Welsh L. Signal transduction by vascular endothelial growth factor receptors. Biochem Soc Trans 2003;31:20-24.

33 Bourette R, Myles G, Choi J, et al. Sequential activation of phoshatidylinositol 3-kinase and phospholipase C-gamma2 by the M-CSF receptor is necessary for differentiation signaling. EMBO J 1997;16:5880-5893.

34 Cao Y, Baig M, Hamm L, et al. Growth factors stimulate kidney proximal tubule cell migration independent of augmented tyrosine phosphorylation of focal adhesion kinase. Biochem Biophys Res Commun 2005;328: 560-566.

35 Feng J, Witthuhn B, Matsuda T, et al. Activation of Jak2 catalytic activity requires phosphorylation of Y1007 in the kinase activation loop. Mol Cell Biol 1997;17: 2497-2501.

36 Feshchenko E, Langdon W, Tsygankov A. Fyn, Yes, and Syk phosphorylation sites in c-Cbl map to the same tyrosine residues that become phosphorylated in activated T cells. J Biol Chem 1998;273:8323-8331.

37 He Y, Wertheim J, Xu L, et al. The coiled-coil domain and Tyr177 of bcr are required to induce a murine chronic myelogenous leukemia-like disease by bcr/abl. Blood 2002;99:2957-2968.

38 Kwon Y, Bhattacharyya A, Alberta J, et al. Activation of ErbB2 during wallerian degeneration of sciatic nerve. J Neurosci 1997;17:8293-8299.

39 Mohammadi M, Dikic I, Sorokin A, et al. Identification of six novel autophosphorylation sites on fibroblast growth factor receptor 1 and elucidation of their importance in receptor activation and signal transduction. Mol Cell Biol 1996;16:977-989.

40 Panayotou G, Bax B, Gout I, et al. Interaction of the p85 subunit of PI 3-kinase and its N-terminal SH2 domain with a PDGF receptor phosphorylation site: structural features and analysis of conformational changes. EMBO J 1992;11:4261-4272.

41 van der Geer P, Wiley S, Gish G, et al. The Shc adaptor protein is highly phosphorylated at conserved, twin tyrosine residues (Y239/240) that mediate proteinprotein interactions. Curr Biol 1996;6:1435-1444.

42 Zrihan-Licht S, Fu Y, Settleman J, et al. RAFTK/Pyk2 tyrosine kinase mediates the association of p190 RhoGAP with RasGAP and is involved in breast cancer cell invasion. Oncogene 2000;19:1318-1328. 